\title{
High mobility group box 1 induces calcification of aortic valve interstitial cells via toll-like receptor 4
}

\author{
WENJUN SHEN $^{1}$, JIANQING ZHOU ${ }^{1}$, CHAOYANG WANG ${ }^{1}$, GUANGZE XU $^{1}$, YING WU ${ }^{1}$ and ZHAOHUI HU $^{2}$ \\ ${ }^{1}$ Ningbo Medical Treatment Center, Lihuili Hospital, Ningbo, Zhejiang 310041; \\ ${ }^{2}$ Department of Cardiovascular Disease, The Affiliated Tongji Hospital, Tongji University, Shanghai 210062, P.R. China
}

Received November 15, 2015; Accepted November 15, 2016

DOI: $10.3892 / \mathrm{mmr} .2017 .6287$

\begin{abstract}
Chronic inflammation and the calcification of aortic valve interstitial cells (AVICs) are the primary etiologies of calcific aortic valve disease (CAVD). However, the underlying mechanism remains to be elucidated. The present study investigated the importance of high mobility group box 1 (HMGB1) via toll-like receptor 4 (TLR4) for the regulation of inflammation and calcification in AVICs. It was determined that the expression levels of HMGB1 and TLR4 were increased in the calcific region of aortic valves with CAVD. In cultured primary AVICs from wild-type mice, HMGB1 treatment demonstrated a dose-dependent increase in mineralization levels and osteogenic gene expression. These effects were significantly reduced in AVICs obtained from TLR4 knockout mice $\left(\mathrm{TLR}^{-/}{ }^{-/}\right.$). In addition, calcification was inhibited by TLR4-specific antibodies in primary AVICs. HMGB1 induced the activation of p38 and nuclear factor $-\kappa \mathrm{B}(\mathrm{NF}-\kappa \mathrm{B})$ in $\mathrm{TLR} 4^{-/-}$primary AVICs, and inhibited p38 and NF- $\kappa$ B in wild-type AVICs treated with TLR4-specific antibodies. The present study demonstrated that TLR4 may function as an essential mediator of HMGB1-induced calcification and in the activation of $\mathrm{p} 38$ and NF- $\mathrm{NB}$.
\end{abstract}

\section{Introduction}

Calcific aortic valve disease (CAVD) is a slow progressive pathologic process that exhibits mild thickening of the aortic valve and calcification of valve leaflets (1). CAVD may be an actively regulated process that includes chronic inflammation, lipoprotein deposition, the renin-angiotensin system, extracellular matrix remodeling, the activation of specific osteogenic signaling pathways and apoptosis. However, degeneration of the aortic valve is the key etiology of CAVD (occurring in $81.9 \%$ of

Correspondence to: Dr Zhaohui Hu, Department of Cardiovascular Disease, The Affiliated Tongji Hospital, Tongji University, 389 Village Road, Shanghai 210062, P.R. China

E-mail: zhaohuihu2006@wo.cn

Key words: high mobility group box 1, toll-like receptor 4, calcification, calcific aortic valve disease, aortic valve interstitial cells
CAVD patients) (2), and specific osteogenic signaling pathways determine the activation and differentiation of the resident fibroblasts or quiescent valve interstitial cells (VICs) into myofibroblasts (activated VICs) and osteoblast-like cells (osteoblastic VICs) with subsequent micro- and macro-calcification (3-5). Therefore, inflammatory responses participate in all stages of CAVD and promote its pathophysiological progression $(6,7)$.

High mobility group box 1 (HMGB1) is a non-histone DNA binding protein (8). HMGB1 is involved in the stabilization of DNA and promotion of gene transcription (9). However, previous studies have demonstrated that HMGB1 participates in inflammation through effects on macrophages $(10,11)$. The expression level of HMGB1 was demonstrated to be increased in acute and chronic inflammatory diseases, such as type 2 diabetes (12), chronic asthma (13) and chronic rhinosinusitis (14). HMGB1 signals through receptor of advanced glycation end products, toll-like receptor (TLR) 2 and TLR4, thereby stimulating an inflammatory response in cells (15). However, the role of HMGB1 in CAVD remains to be elucidated.

A previous study demonstrated that TLR4 proteins are highly expressed in human aortic valve leaflets and aortic valve interstitial cells (AVICs) (16). Stimulation with LPS may result in the activation of the nuclear factor $-\kappa \mathrm{B}(\mathrm{NF}-\kappa \mathrm{B})$ signaling pathway (17). The TLR4 signaling pathway has been demonstrated to enhance the pro-osteogenic response in AVICs and may promote the progression of CAVD (18).

Chronic inflammation is crucial for the development of CAVD, as it was observed to promote the osteoblastic differentiation of AVICs (19). The present study used immunohistochemistry to demonstrate that HMGB1 and TLR4 were expressed in the aortic valve of CAVD samples. In addition, it was revealed that HMGB1 is important for osteoblastic differentiation in AVICs, which is mediated by TLR4. TLR4 was demonstrated to be essential for HMGB1-induced runt-related transcription factor 2 (Runx2), msh homeobox 2 (Msx2), bone morphogenetic protein 2 (BMP2) and osteopontin (OPN) expression in AVICs. These findings provide a novel mechanism for CAVD progression and improve the understanding of aortic valve disease.

\section{Materials and methods}

Reagents and antibodies. HMGB1 (R\&D Systems, Inc., Minneapolis, MN, USA) was used to stimulate AVICs. The 
antibodies used to detect HMGB1 (catalog no. ab18256) and TLR4 (catalog no. ab13556) by immunohistochemical analysis were purchased from Abcam (Cambridge, UK). The 3,3'-Diaminobenzidine Liquid Substrate system, Alkaline Phosphatase Diethanolamine Activity kit and alizarin red S stain were purchased from Sigma-Aldrich; Merck Millipore (Darmstadt, Germany). Dulbecco's modified Eagle's medium (DMEM): F12, supplemented with $20 \%$ fetal bovine serum (FBS), $100 \mathrm{U} / \mathrm{ml}$ penicillin and $100 \mu \mathrm{g} / \mathrm{l}$ streptomycin, was obtained from Gibco; Thermo Fisher Scientific, Inc. (Waltham, MA, USA). The primary antibodies used were specific to Runx2 (catalog no. ab23981), Msx2 (catalog no. ab69058), BMP2 (catalog no. ab14933), OPN (catalog no. ab91655; Abcam), $\beta$-actin (catalog no. 4970S; Cell Signaling Technology, Inc., Danvers, MA, USA), p38 mitogen-activated protein kinase (MAPK; catalog no. 8690S; Cell Signaling Technology, Inc.), phosphorylated (p)-p38-MAPK (p-p38-MAPK; catalog no. 9215S; Cell Signaling Tecnhology, Inc.), NF-кB (catalog no. 8242S; Cell Signaling Technology, Inc.) and p-NF- $\mathrm{B}$ (catalog no. 3033S; Cell Signaling Technology, Inc.). SignalStain ${ }^{\circledR}$ Boost Detection reagent (catalog no. 8114; Cell Signaling Technology, Inc.) was used to detect primary antibodies. Activation of TLR4 was inhibited using an anti-mouse TLR4/MD-2 antibody (cat. no. 16-9924; eBioscience, Inc., San Diego, CA, USA), and a mouse IgG isotype control antibody (catalog no. 16-4714; eBioscience, Inc.) was used as a negative control.

Clinical samples. Human aortic valves with calcific regions were obtained from 3 patients who underwent a valve replacement surgery. Normal aortic valve leaflets were collected from the explanted hearts of 3 patients undergoing heart transplantation surgery. All patients were admitted to The Affiliated Tongji Hospital (Shanghai, China) between August 2015 and December 2016. All patients were male (weight, $65 \pm 5 \mathrm{~kg}$; age, $70 \pm 3$ years). The protocols of the present study were approved by the Ethics Committee of Tongji Hospital, Shanghai Tongji University School of Medicine (Shanghai, China) and written informed consent was obtained from all patients.

Primary AVIC culture. Male, 6-week-old (weight, 10-15 g) wild-type (C57BL/6; n=20) and TLR4 knockout mice $\left(\mathrm{TLR} 4^{-/-} ; \mathrm{n}=20\right)$ were obtained from the Model Animal Research Center of Nanjing University (Nanjing, China). To avoid the stress response, the animals had free access to water and a normal diet for 2 weeks and housed at 5 mice per cage, under a 12-h light/dark cycle at room temperature. The mice were anesthesized with $100 \mathrm{mg} / \mathrm{kg}$ sodium pentobarbital and their aortic valves were obtained for the culture of primary AVICs using the methods described previously (20). Valve leaflets were subjected to collagenase digestion and gently scraped to expose the endothelial layer. The leaflets were then divided into 1-2 $\mathrm{mm}^{2}$ pieces and cultured in DMEM: F12 (1:1) supplemented with 20\% FBS, 2 mmol/l L-glutamine (Gibco; Thermo Fisher Scientific, Inc.), $100 \mathrm{U} / \mathrm{ml}$ penicillin and $100 \mu \mathrm{g} / 1$ streptomycin. The cells were cultured in $5 \% \mathrm{CO}_{2}$ at $37^{\circ} \mathrm{C}$. When $80 \%$ confluence was reached, AVICs were passaged using $0.25 \%$ trypsin-EDTA. AVICs at passages 3 and 8 were used for further experiments. The animal experimental procedures employed in the current study complied with the
Animal Management Rules of the Chinese Ministry of Health (Document no. 55, 2001), and were approved by the Animal Care Committee of Tongji University (Shanghai, China).

Immunohistochemistry. Human calcific $(\mathrm{n}=3)$ and non-calcific aortic valves $(n=3)$ were used for histological and immunochemical analysis. The samples were fixed in $4 \%$ paraformaldehyde overnight and divided into serial $5-\mu \mathrm{m}$ cryosections. The sections were stained with $5 \%$ hematoxylin and $0.5 \%$ eosin for $15 \mathrm{~min}$ at room temperature. Immunohistochemical analysis was performed using anti-HMGB1 (dilution, 1:50) and anti-TLR4 (dilution, 1:50) antibodies at room temperature for $2 \mathrm{~h}$. Following incubation with HRP-conjugated secondary antibodies (dilution, 1:100) at room temperature for $1 \mathrm{~h}$, the sections were incubated with 3,3'-diaminobenzidine (catalog no. D8001; Sigma-Aldrich; Merck Millipore). The expression levels of HMGB1 and TLR4 detected by immunohistochemistry were determined using Image Pro-Plus software version 6.0 (Media Cybernetics, Inc., Rockville, MD, USA). The results were determined as integrated optical density/area.

In vitro calcification of AVICs. Primary AVICs were isolated from the aortic valves of C57BL/6 and TLR $4^{-/-}$knockout mice. AVIC calcification was induced in an osteogenic medium containing DMEM, supplemented with $15 \% \mathrm{FBS}, 50 \mathrm{mg} / \mathrm{ml}$ ascorbate-2-phosphate, $10 \mathrm{nM}$ dexamethasone and $10 \mathrm{mM}$ $\beta$-glycerol phosphate (all purchased from Gibco; Thermo Fisher Scientific) as described previously (21). The culture medium was refreshed every 48-72 h, and the cells were harvested after 3 weeks. The extent of calcification of AVICs was determined by alizarin red $\mathrm{S}$ staining. The cells were washed in distilled water and then exposed to freshly prepared $2 \%$ alizarin red $\mathrm{S}(\mathrm{pH} 4.1-4.3)$ for $5 \mathrm{~min}$, where a red/orange color indicated positive staining. For quantitative analysis of alizarin red $\mathrm{S}$ staining, the dye was released from the cell matrix by incubating the cells with cetylpyridinium chloride for $15 \mathrm{~min}$ at room temperature. The quantity of released dye was determined using a spectrophotometer at $540 \mathrm{~nm}$. Alkaline phosphatase (ALP) activity was determined using spectrophotometry to quantify the level of the p-nitrophenol in AVICs, according to the methods described previously (22). The quantity of alizarin red S staining and ALP activity were normalized to the total quantity of cellular protein using Image Pro-Plus software version 6.0. The quantity of cellular protein was measured by Bichinchoninic Acid assay.

Inhibition of TLR4 activation. Primary AVICs from C57BL/6 mice were seeded onto 6-well plates at a density of $5 \times 10^{6}$ cells/well, before they were pretreated with $5 \mu \mathrm{g} / \mathrm{ml}$ anti-TLR4 antibody (1:20) or $5 \mu \mathrm{g} / \mathrm{ml}$ mouse $\operatorname{IgG}(1: 20)$ as a negative control for $1 \mathrm{~h}$, and subsequently stimulated with HMGB1 at concentrations of $0.5,1$ or $2 \mu \mathrm{g} / \mathrm{ml}$ for $60 \mathrm{~min}$ or 3 weeks.

Western blotting. AVICs $\left(5 \times 10^{6}\right.$ cells) were lysed with the ProteoJET Mammalian Cell Lysis reagent (Fermentas; Thermo Fisher Scientific, Inc.) to extract cytoplasmic proteins. Equal quantities of protein $(50 \mu \mathrm{g})$ were subjected to $10 \%$ SDS-PAGE and blotted onto polyvinylidene difluoride membranes. The membrane was blocked with $5 \%$ bovine 
serum albumin (catalog no. V900933; Sigma-Aldrich; Merck Millipore) and probed with antibodies against TLR4 (dilution, 1:500), Runx2 (dilution, 1:1000), Msx2 (dilution, 1:1,000), BMP2 (dilution, 1:1,000), OPN (dilution, 1:1,000), $\beta$-actin (dilution, 1:2,000), p38-MAPK (dilution, 1:1,000), p-p38-MAPK (dilution, 1:1,000), NF- $\kappa \mathrm{B}$ (dilution, 1:1,000) and $\mathrm{p}-\mathrm{NF}-\kappa \mathrm{B}$ (dilution, $1: 1,000$ ) overnight at $4^{\circ} \mathrm{C}$, followed by incubation with HRP-conjugated secondary antibodies (dilution, 1:5,000) for $1 \mathrm{~h}$ at room temperature. The blots were developed using an enhanced chemiluminescence detection system (Merck Millipore). Images were captured, and the intensity of each band was analyzed with Quantity One software version 4.62 (Bio-Rad Laboratories, Inc., Hercules, CA, USA).

Statistical analysis. Data are expressed as the mean \pm standard deviation. Student's paired $t$-test was performed to compare paired samples and analysis of variance was used for multiple group comparisons, followed by Friedman's post-hoc test. $\mathrm{P}<0.05$ was considered to indicate statistically significant difference. Statistical analyses were performed using SPSS software version 20 (IBM SPSS, Armonk, NY, USA).

\section{Results}

Calcific aortic valves exhibit HMGB1 and TLR4 expression. To investigate the pathology of calcific aortic valves, calcific $(n=3)$ and non-calcific aortic valves $(n=3)$ were stained with HE. Routine pathological examination of calcific aortic valves revealed calcification, macrophage rupture and AVIC thickening close to the region of calcification (Fig. 1A). In calcific aortic valves, the positive expression of HMGB1 and TLR4 was detected across the region of calcification and the integrated optical density/area level of HMGB1 and TLR4 was significantly higher when compared with those in the control aortic valve group (HMGB1, 78.1 \pm 7.6 and TLR4, 84.2 \pm 7.3 in calcific aortic valves vs. HMGB1, 25.1 \pm 3.4 and TLR4, $28.0 \pm 2.4$ in non-calcific aortic valves; $\mathrm{P}<0.01$; Fig. 1B). These results indicated that HMGB1 and TLR4 may contribute to the calcification of the aortic valves.

HMGB1 induces AVIC calcification. The present study investigated whether HMGB1 was involved in the calcification of AVICs in mice. Primary AVICs were stimulated with HMGB1 in order to assess its effects on the calcification of AVICs and the expression levels of Runx2, Msx2, BMP2 and OPN. The ALP activity and alizarin red S staining assays demonstrated that HMGB1 treatment promoted calcification in a dose-dependent manner (Fig. 2A and B). The levels of ALP activity and alizarin red $S$ staining increased significantly at concentrations of 1 and $2 \mu \mathrm{g} / \mathrm{ml}$ HMGB1 when compared with the control group (ALP activity, $1 \mu \mathrm{g} / \mathrm{ml}$ HMGB1, $434.32 \pm 34.51$ vs. $285.33 \pm 34.95, \mathrm{P}<0.01$ and $2 \mu \mathrm{g} / \mathrm{ml}$ HMGB1, $510.34 \pm 29.28$ vs. $285.33 \pm 34.95, \mathrm{P}<0.01$; alizarin red $\mathrm{S}$ staining assay, $1 \mu \mathrm{g} / \mathrm{ml} \mathrm{HMGB} 1,148.21 \pm 7.43$ vs. $114.33 \pm 17.50, \mathrm{P}<0.05$ and $2 \mu \mathrm{g} / \mathrm{ml} \mathrm{HMGB1}, 174.67 \pm 9.03$ vs. $114.33 \pm 17.50, \mathrm{P}<0.01$; Fig. 2A and B). Runx2, Msx2, BMP2 and OPN have been previously demonstrated to be involved in and promote the calcification of AVICs (23). The present study used western blotting to determine the effects of HMGB1 on their protein expression levels. Following exposure of AVICs to HMGB1,
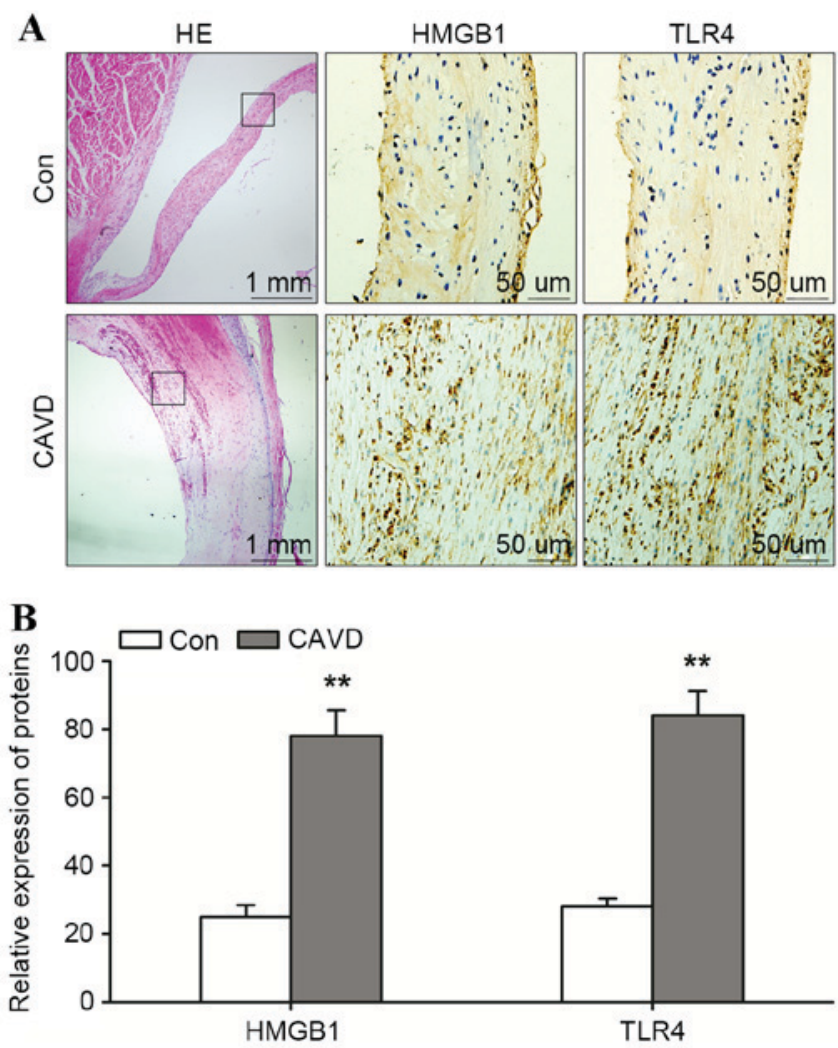

Figure 1. Expression of HMGB1 and TLR4 in the calcification region of CAVD arterial valves. Human aortic valves with calcific and normal aortic valve leaflets were collected from explanted hearts of patients and were assessed by histological and IHC analyses. (A) Sections were stained with HE or with specific antibodies against HMGB1 and TLR4. The black boxes represent the area presented in the IHC staining images. (B) The expression levels of HMGB1 and TLR4 were detected by IHC and were quantified using Image Pro-Plus version 6.0. Data are presented as integrated optical density/area. Three different sections and five different fields of view were analyzed for each section $(n=3) .{ }^{* *} \mathrm{P}<0.01$ vs. Con. HMGB1, high mobility group box 1; TLR4, toll-like receptor 4; CAVD, calcific aortic valve disease; IHC, immunohistochemistry; HE, hematoxylin and eosin; Con, control.

the protein expression levels of Runx2, Msx2, BMP2 and OPN demonstrated a dose-dependent increase (Fig. 2C). High doses (1 and $2 \mu \mathrm{g} / \mathrm{ml}$ ) of HMGB1 significantly induced the expression of these genes $(\mathrm{P}<0.01$; Fig. 2D). These results indicated that, HMGB1 upregulated calcification and the expression of associated genes in primary AVICs in a dose-dependent manner, which suggests that HMGB1 may contribute to the calcification process in AVICs.

TLR4 mediates HMGB1-induced calcification of AVICs. In order to determine whether TLR4 mediated the HMGB1-induced calcification in AVICs, as well as the expression levels of associated genes in AVICs, the calcification and protein expression levels of Runx2, Msx2, BMP2 and OPN following HMGB1 treatment in primary AVICs derived from the valves of wild-type (C57BL/6) or TLR4 ${ }^{-/}$mice, were compared. A TLR4 assay was used in order to identify the phenotype of wild-type and TLR $4^{-1}$. TLR4 protein expression was detected in the wild-type, but not in TLR $4^{-\digamma}$-derived AVICs (Fig. 3A). As $2 \mu \mathrm{g} / \mathrm{ml}$ HMGB1 induced the highest calcification in AVICs (Fig. 2A and B), this concentration was used for further experiments. Primary AVICs were 

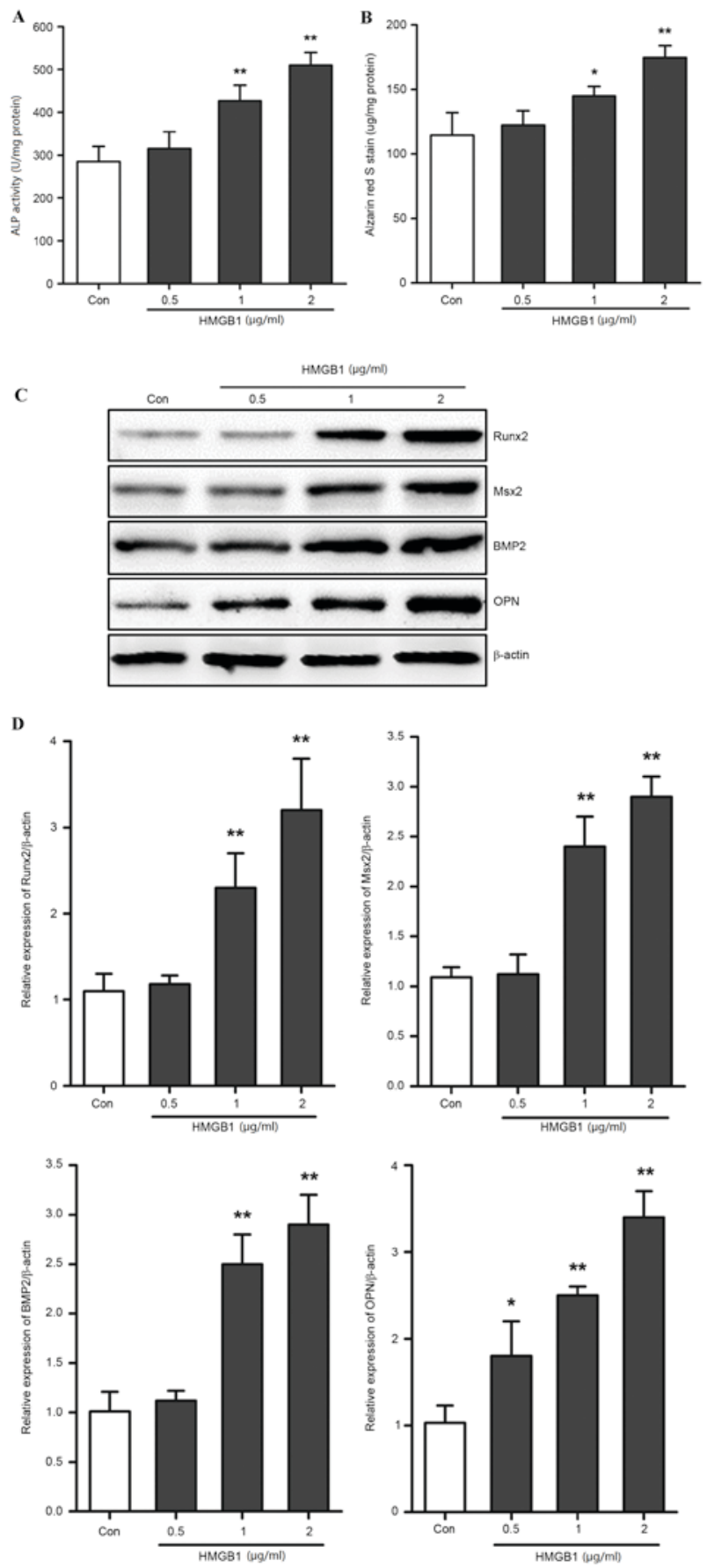

Figure 2. HMGB1 induced calcification of AVICs. Primary AVICs were incubated with $0.5,1$ and $2 \mu \mathrm{g} / \mathrm{ml} \mathrm{HMGB1}$ for 3 weeks. Quantification of (A) ALP activity and (B) alizarin red S staining in AVICs. (C) Western blotting was used to determine the protein expression levels of Runx2, Msx2, BMP2 and OPN. (D) Quantification of band densities by densitometry in 3 independent experiments relative to $\beta$-actin $(n=3)$. ${ }^{*} \mathrm{P}<0.05$ and ${ }^{* *} \mathrm{P}<0.01$ vs. Con. HMGB1, high mobility group box 1 ; AVICs, aortic valve interstitial cells; ALP, alkaline phosphatase; Runx2, runt-related transcription factor 2; Msx2, msh homeobox 2; BMP2, bone morphogenetic protein 2; OPN, osteopontin; Con, control.

incubated with $2 \mu \mathrm{g} / \mathrm{ml}$ HMGB1 for 3 weeks. TLR4 $4^{-/}$AVICs exhibited a significant reduction in ALP activity and alizarin red $\mathrm{S}$ staining when compared with wild-type AVICs $(\mathrm{P}<0.01$; Fig. 3B and C). In addition, the protein expression levels of Runx2, Msx2, BMP2 and OPN, were significantly reduced in TLR $4{ }^{-1}$-derived AVICs when compared with the wild-type
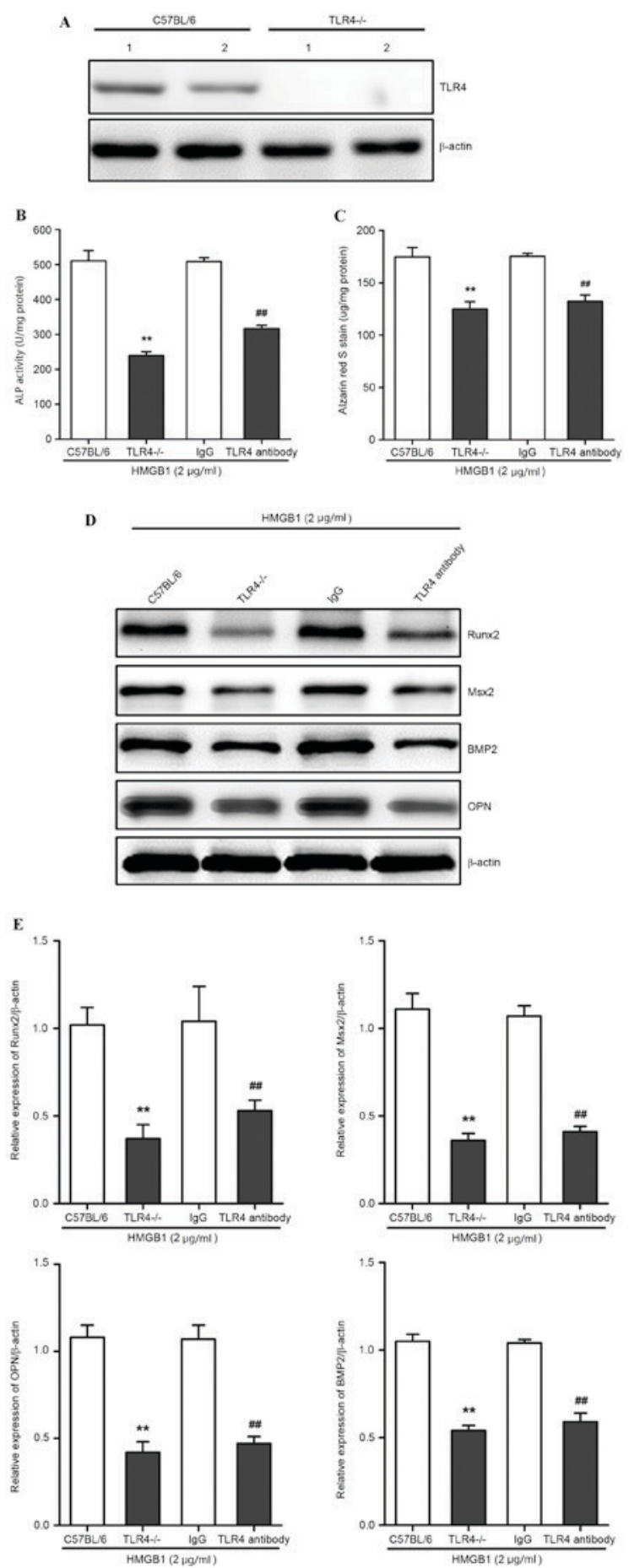

Figure 3. TLR4 mediates HMGB1-induced calcification in AVICs. Primary AVICs were derived from TLR $4^{-/}$and C57BL/6 mice. They were subsequently pretreated with a TLR4-specific antibody, and a mouse IgG was used as a control. (A) TLR4 expression in TLR $4^{-/-}$and C57BL/6-derived AVICs was determined using western blotting ( $n=4$ per genotype). Quantification of (B) ALP activity and (C) alizarin red S staining. (D) Western blotting was used to determine the protein expression levels. (E) Quantification of western blotting by densitometry in 3 independent experiments relative to $\beta$-actin $(\mathrm{n}=3) .{ }^{* *} \mathrm{P}<0.01$ vs. C57BL/6 group; ${ }^{\# \#} \mathrm{P}<0.01$ vs. the mouse IgG treatment group. TLR4, toll-like receptor 4; HMGB1, high mobility group box 1; AVICs, aortic valve interstitial cells; ALP, alkaline phosphatase; Runx 2, runt-related transcription factor 2; Msx2, msh homeobox 2; BMP2, bone morphogenetic protein 2; OPN, osteopontin.

AVICs ( $\mathrm{P}<0.01$; Fig. 3D and E), suggesting that TLR4 may be important for HMGB1-mediated calcification of AVICs. The 
A

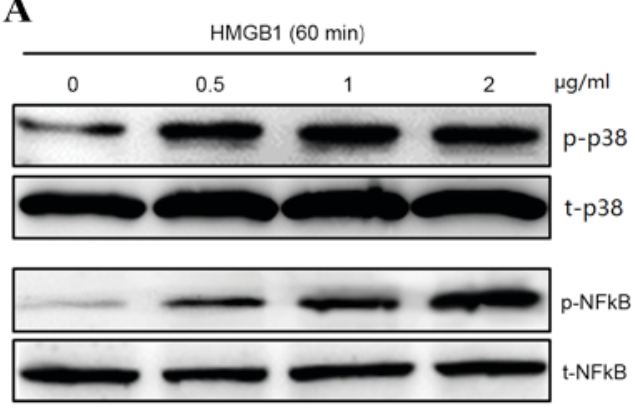

C

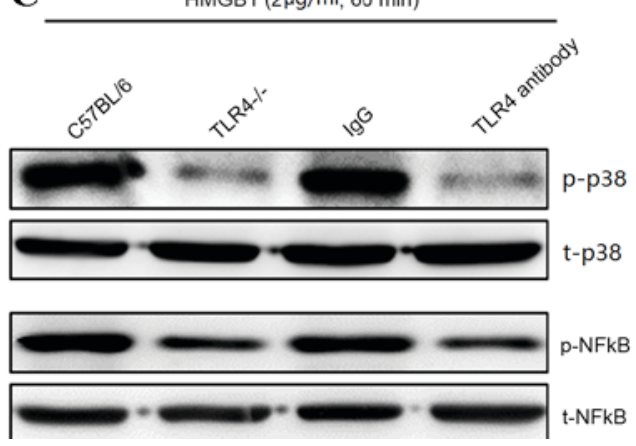

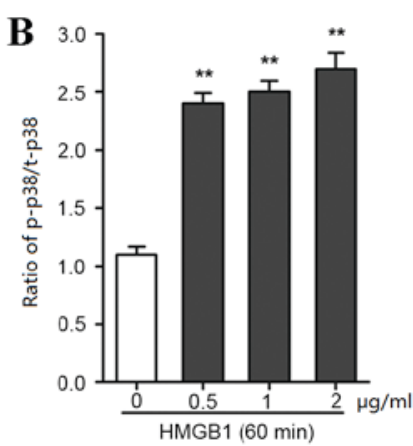

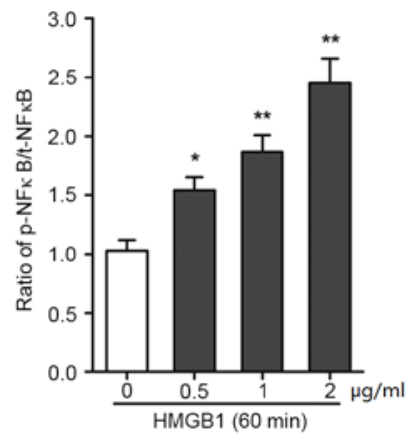

D

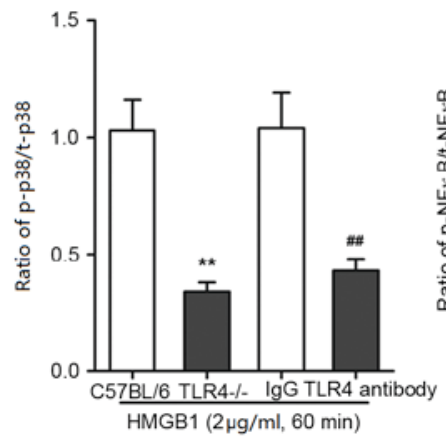

Figure 4. HMGB1 activates p38 and NF- $\kappa$ B in AVICs via TLR4 signaling. Primary AVICs were incubated with $0.5,1$ and $2 \mu \mathrm{g} / \mathrm{ml} \mathrm{HMGB} 1 \mathrm{for} 60 \mathrm{~min}$. The group without HMGB1 treatment was used as a control. Primary AVICs derived from TLR4 ${ }^{-/}$mice or C57BL/6 mice, and C57BL/6-derived AVICs treated with a specific anti-TLR4 antibody (or mouse IgG as the control), were treated with $2 \mu \mathrm{g} / \mathrm{ml} \mathrm{HMGB} 1$ for 60 min to determine the activation of $\mathrm{p} 38$ or NF- $\kappa \mathrm{B}$. The phosphorylation of $\mathrm{p} 38$ and $\mathrm{NF}-\kappa \mathrm{B}$ was detected by (A and C) western blotting and (B and D) quantified by densitometry in 3 independent experiments. ${ }^{*} \mathrm{P}<0.05$ and ${ }^{* *} \mathrm{P}<0.01 \mathrm{vs} .0 \mu \mathrm{g} / \mathrm{ml}$ HMGB1 control. The results are expressed as relative units ( $\mathrm{p} 38$ or NF- $\mathrm{BB}$ phosphorylated protein/total protein). ${ }^{* *} \mathrm{P}<0.01 \mathrm{vs}$. the C57BL/6 group; ${ }^{\# \#} \mathrm{P}<0.01$ vs. the IgG group. HMGB1, high mobility group box 1; AVICs, aortic valve interstitial cells; p-p38, phosphorylated-p38; t-p38,

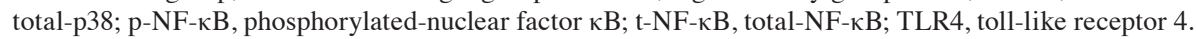

activation of TLR4 was then inhibited using TLR4-specific antibodies, as described previously (24). TLR4-specific antibodies significantly inhibited the increase in HMGB1-induced calcification in primary AVICs from C57BL/6 mice, as demonstrated by the significant reduction in ALP activity and alizarin $S$ staining when compared with the mouse IgG control $(\mathrm{P}<0.01$; Fig. 3B and $\mathrm{C})$. The protein expression levels of Runx2, Msx2, BMP2 and OPN in the TRL antibody group were significantly reduced when compared with the mouse IgG control $(\mathrm{P}<0.01$; Fig. 3D and $\mathrm{E})$. These findings demonstrated that HMGB1-induced calcification was significantly attenuated following TLR4 knockout and inhibition, which suggests that AVIC calcification in response to HMGB1stimulation may require TLR4.

$H M G B 1$ activated $p 38$ and $N F-\kappa B$ via TLR4 in AVICs. The authors hypothesized that TLR4 may interact with MAPK in AVICs following exposure to HMGB1. The phosphorylation of p38 was significantly increased following HMGB1 treatment at concentrations of $0,0.5,1$ and $2 \mu \mathrm{g} / \mathrm{ml}$ for $60 \mathrm{~min}$ $(\mathrm{P}<0.01$; Fig. 4A and B). Notably, the phosphorylation of $\mathrm{NF}-\kappa \mathrm{B}$, as the downstream effect of p38 phosphorylation, was significantly increased following $60 \mathrm{~min}$ incubation with $0,0.5,1$ and $2 \mu \mathrm{g} / \mathrm{ml}$ HMB1 (P<0.01; Fig. 4A and B). To determine whether the HMGB1-induced activation of p38 MAPK was dependent on TLR4 in AVICs, specific anti-TLR4 antibodies and TLR4 ${ }^{-/}$-derived AVICs were used to reduce TLR4 expression levels and activity. TLR4 knockout and inhibition significantly decreased p38 and NF- $\kappa \mathrm{B}$ activation in AVICs when compared with the control treatments $(\mathrm{P}<0.01$; Fig. $4 \mathrm{C}$ and $\mathrm{D})$. These findings suggested that the molecular mechanism underlying HMGB1 treatment may involve TLR4 and p38 MAPK to mediate inflammatory responses.

\section{Discussion}

Chronic inflammation is involved in CAVD and may induce AVIC calcification (25). HMGB1, as an inflammatory cytokine, may contribute to chronic inflammatory responses (12-14). The results of the current study demonstrated that the expression of HMGB1 was increased in the calcification region of CAVD aortic valves, which suggests that HMGB1 may contribute to the calcification of AVICs. AVICs were then incubated with different doses of HMGB1, and analysis of ALP activity and alizarin red S staining levels demonstrated a dose-dependent increase. Wang et al (26) determined that diabetes accelerated saphenous vein graft calcification by $\geq 2$ years following coronary artery bypass grafting surgery; however, HMGB1 mediated the high-glucose-induced calcification in vascular smooth muscle cells of saphenous veins (24). Lai et al (27) reported that HMGB1 was involved in aortic aneurysms induced by calcium chloride in mice. These results demonstrate that HMGB1 may function as a key factor that enhances AVIC calcification. The results of the present study demonstrated 
that HMGB1 promoted the expression of Runx2, Msx2, BMP2 and OPN in a dose-dependent manner. Runx2, Msx2, BMP2 and OPN are important genes associated with calcification. Runx 2 is an osteogenic and chondrogenic transcription factor regulated by post-translational modifications, sub-nuclear localization, and modulatory protein-protein interactions (28). A previous study determined that the expression level of Runx 2 was increased in atherosclerotic calcification and endochondral mineralization programs (29). Hydrogen peroxide was observed to activate osteogenic Runx2 (30) and Msx2/Wnt (31) signaling, thus enhancing mineralization. A previous study indicated that BMP2 increased the secretion of OPN through upregulation of ALP, which led to the degradation of tissue pyrophosphate (32). Runx 2 promoted OPN expression in cardiovascular calcification (33). The present study revealed that HMGB1 was expressed in calcified valves, and that HMGB1 treatment induced calcification of AVICs and osteogenic protein expression. Therefore, the present findings emphasized the importance of HMGB1 in the calcification of CAVD.

The results of the present study demonstrated that TLR4, as a receptor of HMGB1, mediated the extent of the inflammatory response and participated in the pathology of CAVD. Zeng et al $(18,34)$ determined that AVICs from stenotic valves expressed higher levels of BMP2 in response to TLR4 stimulation and NF- $\kappa \mathrm{B}$ activation $(17,33)$. In addition, Deng et al (16) demonstrated that adult AVICs exhibit greater inflammatory and osteogenic responses to TLR4 stimulation (34). Together, these results suggest that the HMGB1-induced mineralization of AVICs may be regulated by TLR4. The results of the present study indicated that the calcification region of aortic valves expressed higher levels of TLR4 and HMGB1 when compared with control valves. ALP activity and the level of alizarin red $\mathrm{S}$ staining were increased by incubating cells with HMGB1; however, these effects were significantly reduced in TLR4 ${ }^{-1-}$ mice and when anti-TLR4 antibodies were used to inhibit TLR4. These findings demonstrate that TLR4 may mediate HMGB1-induced AVIC calcification. Furthermore, the protein expression levels of Runx2, Msx2, BMP2 and OPN were significantly reduced following TLR4 knockout or inhibition. Therefore, TLR4 altered calcification and the expression of a various of osteogenic proteins involved in HMGB1-induced AVIC mineralization.

CAVD is a chronic inflammatory disease where the inflammatory response accelerates calcification (6,7). HMGB1 may induce phosphorylation of $\mathrm{p} 38$ and $\mathrm{NF}-\kappa \mathrm{B}$, thus activating an inflammatory response via TLR4 (15). The present study incubated AVICs with different doses of HMGB1 for $60 \mathrm{~min}$, and the activation of $\mathrm{p} 38$ and $\mathrm{NF}-\kappa \mathrm{B}$ increased in a dose-dependent manner. Additionally, p38 and NF- $\mathrm{B}$ phosphorylation was inhibited following TLR4 knockout or inhibition. These results demonstrated that TLR4 mediated HMGB1-induced p38 and $\mathrm{NF}-\kappa \mathrm{B}$ phosphorylation, thereby increasing the inflammatory response in AVICs. In addition, this suggests that the p38/NF- $\kappa$ B signaling pathway may be a promoter of inflammatory calcification that participates in the pathology of CAVD.

In conclusion, the present study demonstrated the expression of HMGB1 and TLR4 in the calcification region of CAVD arterial valves. Using an in vitro model it was revealed that this activation was functionally and physically associated with
AVIC calcification. The clinical implication of the present study was that deactivation of HMGB1 and TLR4 may effectively attenuate the pathogenesis of CAVD.

\section{References}

1. Freeman RV and Otto CM: Spectrum of calcific aortic valve disease: Pathogenesis, disease progression, and treatment strategies. Circulation 111: 3316-3326, 2005.

2. Iung B, Baron G, Butchart EG, Delahaye F, Gohlke-Bärwolf C, Levang OW, Tornos P, Vanoverschelde JL, Vermeer F, Boersma $\mathrm{E}$, et al: A prospective survey of patients with valvular heart disease in Europe: The Euro heart survey on valvular heart disease. Eur Heart J 24: 1231-1243, 2003.

3. Rajamannan NM, Evans FJ, Aikawa E, Grande-Allen KJ, Demer LL, Heistad DD, Simmons CA, Masters KS, Mathieu P, O'Brien KD, et al: Calcific aortic valve disease: Not simply a degenerative process: A review and agenda for research from the National heart and lung and blood institute aortic stenosis working group. Executive summary: Calcific aortic valve disease-2011 update. Circulation 124: 1783-1791, 2011.

4. Jian B, Narula N, Li QY, Mohler ER III and Levy RJ: Progression of aortic valve stenosis: TGF-betal is present in calcified aortic valve cusps and promotes aortic valve interstitial cell calcification via apoptosis. Ann Thorac Surg 75: 457-466, 2003.

5. Galeone A, Brunetti G, Oranger A, Greco G, Di Benedetto A Mori G, Colucci S, Zallone A, Paparella D and Grano M: Aortic valvular interstitial cells apoptosis and calcification are mediated by TNF-related apoptosis-inducing ligand. Int J Cardiol 169: 296-304, 2013

6. Cotè N, Mahmut A, Bosse Y, Couture C, Pagé S, Trahan S, Boulanger MC, Fournier D, Pibarot P and Mathieu P: Inflammation is associated with the remodeling of calcific aortic valve disease. Inflammation 36: 573-581, 2013.

7. O'Brien KD: Epidemiology and genetics of calcific aortic valve disease. J Investig Med 55: 284-291, 2007.

8. Andersson U, Erlandsson-Harris $\mathrm{H}$, Yang $\mathrm{H}$ and Tracey $\mathrm{KJ}$ : HMGB1 as a DNA-binding cytokine. J Leukoc Biol 72: 1084-1091, 2002.

9. Ueda T, Chou H, Kawase T, Shirakawa H and Yoshida M: Acidic C-tail of HMGB1 is required for its target binding to nucleosome linker DNA and transcription stimulation. Biochemistry 43: 9901-9908, 2004.

10. Yamada S and Maruyama I: HMGB1, a novel inflammatory cytokine. Clin Chim Acta 375: 36-42, 2007.

11. Li L, Ling Y, Huang M, Yin T, Gou SM, Zhan NY, Xiong JX, Wu HS, Yang ZY and Wang CY: Heparin inhibits the inflammatory response induced by LPS and HMGB1 by blocking the binding of HMGB1 to the surface of macrophages. Cytokine 72 : 36-42, 2015.

12. Nativel B, Marimoutou M, Thon-Hon VG, Gunasekaran MK, Andries J, Stanislas G, Planesse C, Da Silva CR, Césari M, Iwema T, et al: Soluble HMGB1 is a novel adipokine stimulating IL-6 secretion through RAGE receptor in SW872 preadipocyte cell line: Contribution to chronic inflammation in fat tissue. PLoS One 8: e76039, 2013.

13. Hou C, Kong J, Liang Y, Huang H, Wen H, Zheng X, Wu L and Chen Y: HMGB1 contributes to allergen-induced airway remodeling in a murine model of chronic asthma by modulating airway inflammation and activating lung fibroblasts. Cell Mol Immunol 12: 409-423, 2015.

14. Min HJ, Kim SJ, Kim TH, Chung HJ, Yoon JH and $\mathrm{Kim} \mathrm{CH}$ : Level of secreted HMGB1 correlates with severity of inflammation in chronic rhinosinusitis. Laryngoscope 125: E225-E330, 2015.

15. Lotze MT and Tracey KJ: High-mobility group box 1 protein (HMGB1): Nuclear weapon in the immune arsenal. Nat Rev Immunol 5: 331-342, 2005.

16. Deng XS, Meng X, Zeng Q, Fullerton D, Mitchell M and Jaggers J: Adult aortic valve interstitial cells have greater responses to toll-like receptor 4 stimulation. Ann Thorac Surg 99: 62-71, 2015.

17. Meng X, Ao L, Song Y, Babu A, Yang X, Wang M, Weyant MJ, Dinarello CA, Cleveland JC Jr and Fullerton DA: Expression of functional Toll-like receptors 2 and 4 in human aortic valve interstitial cells: Potential roles in aortic valve inflammation and stenosis. Am J Physiol Cell Physiol 294: C29-C35, 2008. 
18. Zeng Q, Song R, Ao L, Weyant MJ, Lee J, Xu D, Fullerton DA and Meng X: Notch1 promotes the pro-osteogenic response of human aortic valve interstitial cells via modulation of ERK1/2 and nuclear factor $-\kappa \mathrm{B}$ activation. Arterioscler Thromb Vasc Biol 33: 1580-1590, 2013.

19. Mathieu P, Boulanger MC and Bouchareb R: Molecular biology of calcific aortic valve disease: Towards new pharmacological therapies. Expert Rev Cardiovasc Ther 12: 851-862, 2014.

20. Mohler ER III, Chawla MK, Chang AW, Vyavahare N, Levy RJ, Graham L and Gannon FH: Identification and characterization of calcifying valve cells from human and canine aortic valves. J Heart Valve Dis 8: 254-260, 1999.

21. Mathieu P, Voisine P, Pépin A, Shetty R, Savard N and Dagenais F: Calcification of human valve interstitial cells is dependent on alkaline phosphatase activity. J Heart Valve Dis 14: 353-357, 2005.

22. Osman L, Yacoub MH, Latif N, Amrani M and Chester AH: Role of human valve interstitial cells in valve calcification and their response to atorvastatin. Circulation 114 (1 Suppl): I547-I552, 2006.

23. Towler DA: Molecular and cellular aspects of calcific aortic valve disease. Circ Res 113: 198-208, 2013.

24. Ji Y, Liu J, Wang Z and Liu N: Angiotensin II induces inflammatory response partly via toll-like receptor 4-dependent signaling pathway in vascular smooth muscle cells. Cell Physio Biochem 23: 265-276, 2009.

25. Pasipoularides A: Calcific Aortic valve disease: Part 1-molecular pathogenetic aspects, hemodynamics, and adaptive feedbacks. J Cardiovasc Transl Res 9: 102-118, 2016.

26. Wang Y, Shan J, Yang W, Zheng H and Xue S: High mobility group box 1 (HMGB1) mediates high-glucose-induced calcification in vascular smooth muscle cells of saphenous veins. Inflammation 36: 1592-1604, 2013.

27. Lai CH, Shi GY, Lee FT, Kuo CH, Cheng TL, Chang BI, Ma CY, Hsu FC, Yang YJ and Wu HL: Recombinant human thrombomodulin suppresses experimental abdominal aortic aneurysms induced by calcium chloride in mice. Ann Surg 258: 1103-1110, 2013.
28. Steitz SA, Speer MY, Curinga G, Yang HY, Haynes P, Aebersold R, Schinke T, Karsenty G and Giachelli CM: Smooth muscle cell phenotypic transition associated with calcification: Upregulation of Cbfa1 and downregulation of smooth muscle lineage markers. Circ Res 89: 1147-1154, 2001.

29. Tyson KL, Reynolds JL, McNair R, Zhang Q, Weissberg PL and Shanahan CM: Osteo/chondrocytic transcription factors and their target genes exhibit distinct patterns of expression in human arterial calcification. Arterioscler Thromb Vasc Biol 23: 489-494, 2003.

30. Byon CH, Javed A, Dai Q, Kappes JC, Clemens TL, Darley-Usmar VM, McDonald JM and Chen Y: Oxidative stress induces vascular calcification through modulation of the osteogenic transcription factor Runx 2 by AKT signaling. J Biol Chem 283: 15319-15327, 2008

31. Lai CF, Shao JS, Behrmann A, Krchma K, Cheng SL and Towler DA: TNFR1-activated reactive oxidative species signals up-regulate osteogenic Msx2 programs in aortic myofibroblasts. Endocrinology 153: 3897-3910, 2012.

32. Steitz SA, Speer MY, McKee MD, Liaw L, Almeida M, Yang H and Giachelli CM: Osteopontin inhibits mineral deposition and promotes regression of ectopic calcification. Am J Pathol 161: 2035-2046, 2002.

33. Sowa AK, Kaiser FJ, Eckhold J, Kessler T, Aherrahrou R, Wrobel S, Kaczmarek PM, Doehring L, Schunkert H, Erdmann J and Aherrahrou Z: Functional interaction of osteogenic transcription factors Runx 2 and Vdr in transcriptional regulation of Opn during soft tissue calcification. Am J Pathol 183: 60-68, 2013.

34. Zeng Q, Song R, Ao L, Xu D, Venardos N, Fullerton DA and Meng X: Augmented osteogenic responses in human aortic valve cells exposed to oxLDL and TLR4 agonist: A mechanistic role of Notch1 and NF-кB interaction. PLoS One 9: e95400, 2014. 